Lombeida García E, Gómez PandoL, Reyes Borja WO,

Triviño Gilces C, Hasang Moran E, Duran Canare PL, Hufana

ARTÍCULO ORIGINAL

Duran D. Densidad de población de Meloidogyne

ORIGINAL ARTICLE

graminicola en plantaciones comerciales de arroz y

comportamiento de líneas avanzadas en la provincia de

Los Ríos, Ecuador. Rev. Soc. cient. Parag. 2021;26(1):64-81.

https://doi.org/10.32480/rscp.2021.26.1.64

Recibido: 20/03/2021. Aceptado: 25/05/2021.

\title{
Densidad de población de Meloidogyne graminicola en plantaciones comerciales de arroz y comportamiento de líneas avanzadas en la provincia de Los Ríos, Ecuador
}

\section{Population density of Meloidogyne graminicola in commercial rice plantations and behavior of advanced lines in the province of Los Ríos, Ecuador}

Emma Lombeida García ${ }^{1,2 *}$ (D) Luz Gómez Pando² (D) Walter Oswaldo Reyes Borja $^{1}$ (D), Carmen Triviño Gilces ${ }^{1}$ (D), Edwin Hasang Moran ${ }^{1}$ (D), Perry Lorraine Duran Canare ${ }^{3}$ (D), Danilda Hufana Duran ${ }^{1,4}$

${ }^{1}$ Universidad Técnica de Babahoyo. Babahoyo, Los Ríos, Ecuador.

${ }^{2}$ Universidad Nacional Agraria. La Molina, Perú.

${ }^{3}$ Philippine Rice Research Institute and Central Luzon State University, Science City of Muñoz. Nueva Ecija, Philippines. ${ }^{4}$ Philippine Carabao Center and Central Luzon State University, Science City of Muñoz. Nueva Ecija, Philippines.

Autor correspondiente: elombeida@utb.edu.ec

Resumen: En este estudio se determinaron las poblaciones de $M$. graminicola en suelo y raíces obtenidas en plantaciones comerciales de arroz en las zonas de Babahoyo y Quevedo del Ecuador. Para estudiar la tolerancia y/o resistencia al nematodo M. graminicola, se utilizaron un total de 50 líneas avanzadas $F_{5}$ de arroz, de las cuales 35 son derivadas de cruces interespecíficos de Oryza sativa L. ssp. japonica x Oryza rufipogon G. (PUYÓN) y 15 líneas provenientes de cruces intraespecíficos de líneas de Oryza sativa L. ssp. japónica, incluyéndose también una variedad comercial de arroz, la INIAP 15, susceptible al ataque de M. graminicola, utilizada como control. La investigación se llevó a cabo en la Facultad de Ciencias Agropecuarias (FACIAG) de la Universidad Técnica de Babahoyo en Ecuador. Las evaluaciones de densidades poblacionales de $M$. graminicola conducidas en raíces y suelo, se realizaron en el Laboratorio de Fitopatología de la FACIAG. 
En base a los resultados obtenidos, se encontraron diferencias significativas en la respuesta entre las 50 líneas avanzadas $\mathrm{F}_{5}$ de arroz y la variedad comercial INIAP 15 a M. graminicola. En las condiciones evaluadas, las líneas 32 (PUYÓN/JP002 P11 - 10 P74), 5 (PUYÓN/JP002 P8 - 20 P13), 31 (PUYÓN/JP002 P11 - 10 P22) y 2 (PUYÓN/JP002 P8 - 20 P14), presentaron una baja densidad poblacional de M. graminicola en $10 \mathrm{~g}$ raíces. Por otro lado, las líneas 24 (PUYÓN/JP002-P8-28 P28), 28 (PUYÓN/JP002P8-32 P33) y 30 (PUYÓN/JP002-P8-28 P28-11), presentaron una baja densidad poblacional de M. graminicola en $100 \mathrm{~cm}^{3}$ de suelo. En cuanto al índice de reproducción, el mayor número de veces que se multiplicó el nematodo fue 25 veces en la línea 7 (PUYÓN/JP002 P8 -28 P8-5) y el menor 2 veces en la línea 32 (PUYÓN/JP002 P11 - 10 P22).

Palabras clave: arroz, líneas avanzadas, Meloidogyne graminicola, Oryza rufipogon, manejo sustentable.

Abstract: Populations of Meloidogyne graminicola in commercial rice plantations in Ecuador and resistance of F5 rice lines derived from interspecific crosses of Oryza sativa L. ssp. japonica x Oryza rufipogon G. (PUYÓN) and from intraspecific crosses from Oryza sativa L. ssp. japonica lines to the M. graminicola were determined. Population density evaluations were conducted on roots and soil of rice sampled from 20 commercial plantation areas while resistance research was assessed under controlled nursery conditions. A total of 50 advanced F5 rice lines were inoculated with 2,500 J2 M. graminicola with a commercial variety, INIAP 15, susceptible to the attack of M. graminicola. Results showed remarkably high incidence of $M$. graminicola in 13 out of 20 investigated plantation areas. Response to M. graminicola among the inoculated 50 advanced Lines F5 showed that four (4) Lines 32 (PUYÓN / JP002 P11 - 10 P74), 5 (PUYÓN / JP002 P8 - 20 P13), 31 (PUYÓN / JP002 P11 - 10 P22) and 2 (PUYÓN / JP002 P8 - 20 P14) presented a low population density of M. graminicola / $10 \mathrm{~g}$ of roots. Analysis on Reproduction index showed that the least number of times the nematode multiplied was 2 times in Line 32 (PUYÓN / JP002 P11 10 P22) while more than 2 times in the other lines. In conclusion, F5 line from interspecific crosses of Oryza sativa L. ssp. japonica x Oryza rufipogon G. (PUYÓN) has quantitative nematode resistance with Line 32 (PUYÓN / JP002 P11-10 P74) had the lowest nematode attack, suggesting that this line is promising for crop production in nematode prevalent areas. Studies assessing the production performance of these F5 rice lines in the very high nematode identified areas and measures to control the prevalence of nematodes are recommended.

Lombeida García E, Gómez PandoL, Reyes Borja WO, Triviño Gilces C, Hasang Moran E, Duran Canare PL, Hufana Duran D. Densidad de población de Meloidogyne graminicola en plantaciones comerciales de arroz y comportamiento de líneas avanzadas en la provincia de Los Ríos, 
Keywords: rice, advanced lines, Meloidogyne graminicola, Oryza rufipogon, sustainable management.

\section{INTRODUCCIÓN}

El arroz es considerado una fuente de calorías importante para la alimentación de una tercera parte de la población mundial. En Ecuador, es uno de los principales productos de abastecimiento familiar básico, se infiere que la producción de arroz debería aumentar permanentemente para satisfacer la demanda nacional(1). Para direccionar el cultivo del arroz hacia la tecnificación y uso del producto en la industria agroalimentaria, se deben realizar gestiones para reducir daños y costos por rentabilidad y practicidad. De esta forma se requiere dejar atrás los sistemas tradicionales y tecnificar el manejo del cultivo. Sin embargo, es necesario eliminar ciertas barreras para iniciar un nuevo proceso de transformación ${ }^{(2,3)}$.

El arroz de la subespecie japónica es una variedad con hojas gruesas de color verde oscuro, macollos resistentes a la oscilación de altura media y se adapta mejor a los climas templados. Esta subespecie tiene una mayor demanda en los mercados japoneses; por lo tanto, hay un aumento del comercio mundial, para satisfacer las necesidades de los distintos mercados y es muy apreciado en la Unión Europea(4).

En Ecuador, existen factores que limitan los rendimientos y por ende los costos de la producción de arroz, los limitantes más relevantes son la falta de tecnificación de los cultivos, falta de sistemas de riego, uso de la semilla reciclada, uso inadecuado de fertilizantes y principalmente la alta incidencia de plagas entre ellas el nematodo agallador Meloidogyne graminicola. En arroz de regadío se han determinado pérdidas de hasta el $72 \%$ de la cosecha con poblaciones de hasta 4000 juveniles / planta de M. graminicola. En consecuencia, puede existir disminución en el rendimiento de este cultivo, lo que produce impactos económicos y sociales negativos( ${ }^{(6)}$.

Se ha identificado resistencia a M. graminicola en el germoplasma de Oryza en líneas de especies de arroz africanas $O$. glaberrima, 0 . longistaminata y $O$. rufipogon, así como también, en algunas variedades locales de Oryza, que tienen resistencia total o parcial a M. graminicola, utilizadas como donantes de resistencia en cruces interespecíficos con arroz asiático(7). El uso de variedades resistentes es un método de control eficaz contra especies de endoparásitos sedentarios como Meloidogyne, que pasa la mayor parte de su ciclo de vida dentro de las raíces( ${ }^{(8)}$. No obstante, en plantas resistentes, los nematodos mueren después de un tiempo de invadir el tejido vegetal y en

\footnotetext{
Lombeida García E, Gómez PandoL, Reyes Borja WO, Triviño Gilces C, Hasang Moran E, Duran Canare PL, Hufana Duran D. Densidad de población de Meloidogyne graminicola en plantaciones comerciales de arroz y comportamiento de líneas avanzadas en la provincia de Los Ríos, 
plantas con tolerancia, se produce poco daño incluso cuando están infectadas por altos niveles del nematodo ${ }^{(9)}$.

Existen estudios que demuestran nuevos alelos para incrementar la variabilidad genética del rendimiento en arroz destacando los provenientes de 20 especies silvestres del género Oryza ${ }^{(5)}$. Se realizaron cruces entre $O$. rufipogon y O. glaberrima con las especies cultivadas Bg 90-2, Oryzica 3 y Caiapó utilizadas como especies recurrentes y las especies silvestres como donantes en el programa de reproducción interespecífica por retro cruzamiento. Las líneas obtenidas del cruce entre Bg 90-2 y O. rufipogon tuvieron un rendimiento superior entre 5 a $25 \%$ sobre la variedad comercial que produjo $7.2 \mathrm{t} / \mathrm{ha}^{-1}$. En cuanto a la calidad del grano, ambos padres presentan baja calidad de grano, pero se obtuvieron líneas con segregación transgresora con el grano fino y translúcido(5).

El Laboratorio de Biotecnología de la Facultad de Ciencias Agrarias de la Universidad Técnica de Babahoyo ubicado en la provincia de Los Ríos en Ecuador, lleva a cabo un Programa de Mejoramiento Genético del Arroz, realizando cruces interespecíficos de Oryza rufipogon G. PUYÓN x Oryza sativa L. ssp. japónica y cruces intraespecíficos de Oryza sativa L. ssp. línea japónica con la finalidad de obtener líneas japónica puras, para ello, el programa selecciono líneas avanzadas con potencial genético y productivo.

El objetivo de la presente investigación fue evaluar la respuesta a Meloidogyne graminicola de líneas avanzadas $\mathrm{F}_{5}$ derivadas de cruzas interespecíficas, para detectar fuentes de tolerancia y resistencia. Por otro lado, en este estudio también se estudió las poblaciones de M. graminicola en muestras de suelo y raíces obtenidas en plantaciones comerciales de arroz en las áreas de Babahoyo y Quevedo para conocer y tener referencia de las tasas poblacionales de nematodos en las áreas arroceras locales.

\section{MATERIALES Y METODOS}

Ubicación de las áreas experimentales: se tomaron muestras de suelo y raíces de plantaciones comerciales de arroz en las áreas de Babahoyo y Quevedo en la provincia de Los Ríos, Ecuador, por otro lado, el estudio de resistencia de líneas avanzadas de arroz F5 se llevó a cabo en la Facultad de Ciencias Agropecuarias de la Universidad Técnica de Babahoyo (UTB) ubicada en el km 71/2 de la vía Babahoyo-Montalvo, cantón Babahoyo, provincia de Los Ríos ${ }^{(10)}$.

Lombeida García E, Gómez PandoL, Reyes Borja WO, Triviño Gilces C, Hasang Moran E, Duran Canare PL, Hufana Duran D. Densidad de población de Meloidogyne graminicola en plantaciones comerciales de arroz y comportamiento de líneas avanzadas en la provincia de Los Ríos, 
Densidad poblacional de $M$. graminicola en muestras de suelo y raíces: se visitaron 20 localidades en las áreas de Babahoyo y Quevedo, en donde se recolecto 20 muestras de suelo y 20 muestras de raíces obtenidas de cultivos comerciales plantaciones de arroz, se extrajo todo el sistema radicular de las plantas muestreadas de aproximadamente 45 días de edad en función a la aplicación del método de extracción "Liquefied-Sifting"(11). Las raíces de cada planta se cortaron en trozos de $1 \mathrm{~cm}$, se pesaron $10 \mathrm{~g}$ y se licuaron durante 20 segundos a baja velocidad. El batido se vació en tres tamices $\mathrm{N}^{\circ} 60(250 \mu \mathrm{m}), 100(150 \mu \mathrm{m})$ y $500(25 \mu \mathrm{m})$ colocados de arriba abajo, los dos primeros tamices se lavaron con una ducha tipo teléfono durante un minuto cada uno. El contenido de nematodos de agua se recogió en los tamices 500, en este contenido se colocó en un vaso de precipitados con ayuda de abeto y se completó hasta $100 \mathrm{~mL}$. Para determinar la presencia de población de nematodos en $100 \mathrm{~mL}$ se homogeneizó la solución de nematodos de agua con una pequeña bomba de aire (para pecera) y se extrajo una alícuota de $2 \mathrm{~mL}$, el conteo se llevó a cabo con la ayuda del estereoscopio usando un contador de verificación y por cálculo matemático se obtuvo la densidad poblacional de nematodos por planta.

Para obtener la densidad poblacional de M. graminicola en suelo, se recolectaron $200 \mathrm{~cm}^{3}$ de suelo del sitio donde se ubicó la planta, esta tierra se homogeneizó y se colocó en una funda de plástico para cada planta individual. En el laboratorio, cada muestra se colocó en una bandeja de plástico, se volvió a mezclar y se midieron $100 \mathrm{~cm}^{3}$ para la extracción de los nematodos para este caso se utilizó el método de "Incubación"(11). Para ello se prepararon dos placas de aluminio superpuestas, de las cuales la primera fue calada y la segunda como base, en el primero se colocó una fina malla plástica y sobre este una hoja de papel frontal. Se colocó la tierra sobre el papel y se le añadió agua común, dejando la muestra en incubación durante tres días. Después de tres días, se retiró la tierra de la primera placa y se recogió el contenido de nematodos de agua en la segunda placa en un vaso de precipitados de $200 \mathrm{~mL}$, en cada vaso se dejó un volumen de $100 \mathrm{~mL}$, retirando el exceso de agua con el uso de un tamiz No. 500 y para determinar la población de nematodos en los $100 \mathrm{~mL}$, se realizó el mismo procedimiento mencionado anteriormente con las raíces.

Nivel de resistencia al nematodo $M$. graminicola en líneas de arroz $F_{5}$ avanzadas en condiciones de vivero: Para esta actividad se utilizaron un total de 50 líneas de arroz $\mathrm{F}_{5}$ avanzadas de las cuales 35 de ellas derivan de cruces interespecíficos de Oryza rufipogon G. (PUYÓN) x Oryza sativa L. ssp.

\footnotetext{
Lombeida García E, Gómez PandoL, Reyes Borja WO, Triviño Gilces C, Hasang Moran E, Duran Canare PL, Hufana Duran D. Densidad de población de Meloidogyne graminicola en plantaciones comerciales de arroz y comportamiento de líneas avanzadas en la provincia de Los Ríos, 
japónica y 15 líneas de cruces intraespecíficos de Oryza sativa L. ssp. Japónica (Tabla 1).

Multiplicación de M. graminicola y obtención de juveniles de la segunda etapa (J2): Utilizando suelo infestado con M. graminicola de una plantación comercial de arroz, se realizó la multiplicación de nematodos a nivel de invernadero. Las semillas de arroz de la variedad INIAP 15 (susceptible a M. graminicola) se sembraron en 50 macetas con dos plantas extraídas de semilleros infestados. En plantas de aproximadamente 70 días de edad, se recolectaron las raíces y se extrajeron las masas de huevos de nematodos para exponerlas en el laboratorio para obtener nematodos juveniles de la segunda etapa (J2). Para contar la población existente, se colocó $1 \mathrm{~mL}$ de inóculo en placas de Petri en un estereomicroscopio. Se obtuvo una población de 2.500 (J2 o infectiva estados) por medio de un cálculo matemático en un volumen de $3 \mathrm{~mL}$, muestra que se utilizó para la inoculación de cada una de las plantas en estudio.

Tabla 1. Líneas avanzadas de arroz F5 utilizadas en el estudio para determinar la resistencia al nematodo M. graminicola.

\begin{tabular}{|c|c|c|c|c|c|}
\hline $\begin{array}{c}\text { Tratam } \\
\text { iento }\end{array}$ & $\begin{array}{l}\text { Líneas de } \\
\text { arroz }\end{array}$ & $\begin{array}{c}\text { Tratam } \\
\text { iento }\end{array}$ & Líneas de arroz & $\begin{array}{c}\text { Tratam } \\
\text { iento }\end{array}$ & Líneas de arroz \\
\hline 1 & $\begin{array}{l}\text { PUYÓN/J } \\
\text { P002 } \\
\text { P8 - } 20 \\
\text { P36 }\end{array}$ & 18 & $\begin{array}{l}\text { PUYÓN/JP002 P8 } \\
\text { - } 31 \text { P7 }\end{array}$ & 35 & $\begin{array}{l}\text { PUYÓN/JP002 } \\
\text { P11 - } 10 \text { P23 }\end{array}$ \\
\hline 2 & $\begin{array}{l}\text { PUYÓN/J } \\
\text { P002 } \\
\text { P8 - } 20 \\
\text { P14 }\end{array}$ & 19 & $\begin{array}{l}\text { PUYÓN/JP002 P8 } \\
-31 \text { P30 }\end{array}$ & 36 & $\begin{array}{l}\text { JP003/JP001 P169 } \\
\text { P23-6-GL }\end{array}$ \\
\hline 3 & $\begin{array}{l}\text { PUYÓN/J } \\
\text { P002 } \\
\text { P8 - } 20 \\
\text { P22 }\end{array}$ & 20 & $\begin{array}{l}\text { PUYÓN/JP002 P8 } \\
-31 \text { P34 }\end{array}$ & 37 & $\begin{array}{l}\text { JP003/JP001 P169 } \\
\text { P17-36-GL }\end{array}$ \\
\hline 4 & $\begin{array}{l}\text { PUYÓN/J } \\
\text { P002 } \\
\text { P8 - } 20 \\
\text { P27 } \\
\text { PUYÓN/I }\end{array}$ & 21 & $\begin{array}{l}\text { PUYÓN/JP002 P8 } \\
\text { - } 28 \text { P35 }\end{array}$ & 38 & $\begin{array}{l}\text { JP003/JP001 P169 } \\
\text { P21-2-GL }\end{array}$ \\
\hline 5 & $\begin{array}{l}\text { P002 } \\
\text { P8 - } 20 \\
\text { P13 }\end{array}$ & 22 & $\begin{array}{l}\text { PUYÓN/JP002 P8 } \\
-28 \text { P25 }\end{array}$ & 39 & $\begin{array}{l}\text { JP003/JP001 P169 } \\
\text { P14-31-GL }\end{array}$ \\
\hline
\end{tabular}

Lombeida García E, Gómez PandoL, Reyes Borja WO, Triviño Gilces C, Hasang Moran E, Duran Canare PL, Hufana Duran D. Densidad de población de Meloidogyne graminicola en plantaciones comerciales de arroz y comportamiento de líneas avanzadas en la provincia de Los Ríos, 
Rev. Soc. cient. Parag. 2021;26(1):64-81

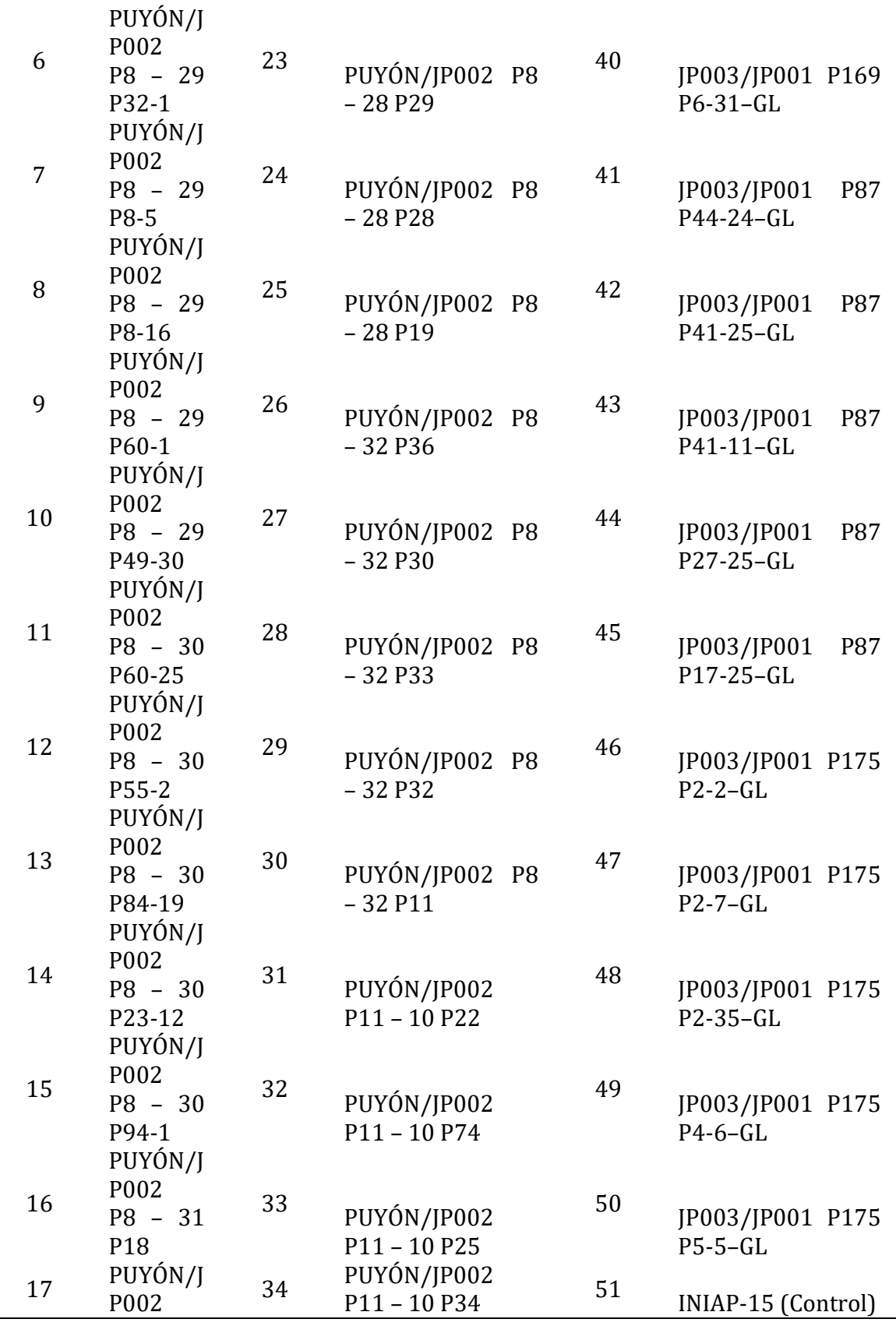

Lombeida García E, Gómez PandoL, Reyes Borja WO, Triviño Gilces C, Hasang Moran E, Duran Canare PL, Hufana Duran D. Densidad de población de Meloidogyne graminicola en plantaciones comerciales de arroz y comportamiento de líneas avanzadas en la provincia de Los Ríos, 
P8 - 31

$\mathrm{P} 4$

Inoculación de líneas avanzadas de arroz $\mathbf{F}_{\mathbf{5}}$ : Se preparó una cantidad de suelo agrícola y se solarizó para dejarlo libre de nematodos y otros microorganismos. Se añadió al sustrato una cantidad adecuada de fertilizantes a base de fósforo y potasio. Se tomó aproximadamente $1 \mathrm{~kg}$ de tierra y se colocó en macetas y se sembraron cinco semillas cada una. Quince días después, las plantas se aclararon dejando dos plantas por maceta. A los 25 días de edad, cada línea avanzada de arroz F5 fue inoculada con una población de 2.500 J2 de M. graminicola. Para la aplicación, se removió una pequeña cantidad de tierra alrededor de las raíces y se depositó lentamente la solución con los juveniles, luego se cubrió las raíces con la misma tierra apartada. Durante 15 días, las plantas se regaron cuidadosamente para evitar perder el inóculo por secado o exceso de humedad del suelo.

Índice de reproducción de $M$. graminicola en líneas de arroz $F_{5}$ avanzadas: El índice de reproducción (IR) de M. graminicola en cada una de las líneas avanzadas de arroz $\mathrm{F}_{5}$ se determinó dividiendo la densidad poblacional del nematodo obtenida de las raíces recolectadas a los 45 días después de la inoculación. o de la denominada población final (Pf) entre la población inicial (Pi) que fueron los 2500 J2 inoculados (RI = Pf / Pi). Para calcular el índice de reproducción y el tipo de hospedante se utilizó la escala determinada por el CIP (1985) (Tabla 2).

Tabla 2. Escala para calificar el índice de reproducción de M. graminicola y determinar el tipo de hospedante

\begin{tabular}{ccc}
\hline Grado & $\begin{array}{c}\text { Indice de reproducción } \\
\mathbf{R I}=\mathbf{P f} / \mathbf{P i}\end{array}$ & Tipo de anfitrión \\
\hline 0 & 0 & No hospedero \\
1 & 0,1 a 0,4 & Hospedero deficiente \\
2 & 0,41 a 1 & Hospedero \\
3 & 1,1 a 2 & Hospedero eficiente \\
4 & $<2$ & Hospedero muy eficiente \\
\hline
\end{tabular}

Fuente: CIP (1985)

Incidencia y severidad de $M$. graminicola en líneas avanzadas $F_{5}$ de arroz: En la Tabla 3, se presenta la escala descrita por Ramos en 1998 (12), para calificar la incidencia y severidad de M. graminicola, para determinar el grado y calificar en función del número de nematodos $/ \mathrm{cm}^{3}$ de suelo y

Lombeida García E, Gómez PandoL, Reyes Borja WO, Triviño Gilces C, Hasang Moran E, Duran Canare PL, Hufana Duran D. Densidad de población de Meloidogyne graminicola en plantaciones comerciales de arroz y comportamiento de líneas avanzadas en la provincia de Los Ríos, 
nematodos / $10 \mathrm{~g}$ de raíces, La calificación de la incidencia del nematodo en plantas de las líneas avanzadas fue: Libre, Baja, Moderada, Alta y Muy alta.

Tabla 3. Escala para calificar la incidencia y severidad de M. graminicola en líneas avanzadas $\mathrm{F}_{5}$ de arroz en condiciones de vivero.

\begin{tabular}{clll}
\hline Grado & $\begin{array}{l}\text { Nematodos/100 } \\
\text { cm }^{\mathbf{3}} \text { de suelo }\end{array}$ & $\begin{array}{l}\text { Nematodos/10 } \\
\text { g de raíces }\end{array}$ & Calificación \\
\hline 0 & 0 & 0 & Libre \\
1 & $1-40$ & 1 a 300 & Baja \\
2 & $41-120$ & 301 a 1000 & Moderada \\
3 & $121-150$ & 1001 a 3000 & Alta \\
4 & $>150$ & $>3000$ & Muy alta \\
\hline
\end{tabular}

Ramos et al.(12).

\section{Diseño experimental y análisis estadístico de los materiales evaluados bajo invernadero}

En este experimento se utilizó el Diseño Completamente al Azar (DCA) con cinco repeticiones y 51 tratamientos (50 líneas avanzadas y 1 variedad comercial). Las diferentes variables que se estudiaron fueron sometidas al Análisis de Varianza (ANOVA) para la determinación de la significancia estadística y a la prueba de Tukey $5 \%$ para diferenciar o comparar los valores de las variables estudiadas. Para realizar el análisis de la varianza se transformaron los valores reales a $\log \mathrm{de} \mathrm{x}$.

\section{RESULTADOS Y DISCUSIÓN}

3.1. Densidad poblacional de $M$. graminicola en muestras de suelo y de raíces, obtenidas en plantaciones comerciales de arroz en las zonas de Babahoyo y Quevedo, provincia de Los Ríos

Las poblaciones de $M$. graminicola observadas en $100 \mathrm{~cm}^{3}$ de suelo, en los 20 sitios de muestreo, se presentan en la tabla 4. Todas las muestras de suelo colectadas en las plantaciones de arroz procedentes de las zonas de Babahoyo y Quevedo presentaron poblaciones de $M$. graminicola. En el cantón Quevedo, las poblaciones de nematodos variaron de 50 a 700/100 $\mathrm{cm}^{3}$ de suelo, con excepción del recinto Guayacán, donde se encontraron 


\section{Rev. Soc. cient. Parag. 2021;26(1):64-81}

1600 J2, calificada como una población muy alta. En la zona de Babahoyo, en la mayor parte de las áreas muestreadas, se encontró un rango de 50 a 300 $\mathrm{J} 2 / 100 \mathrm{~cm}^{3}$ de suelo y en una de las muestras del sector CEDEGE, se registró una población de 600 J2, calificándose como muy alta.

Tabla 4. Densidad poblacional de M. graminicola en $100 \mathrm{~cm}^{3}$ de suelo, procedentes de plantaciones comerciales de arroz de los cantones Babahoyo y Quevedo.

\begin{tabular}{|c|c|c|c|c|c|}
\hline Código & Recinto & Propietario & Zonas & $\begin{array}{l}\text { M. graminicola } \\
/ 100 \mathrm{~cm}^{3} \text { suelo }\end{array}$ & Rangos \\
\hline P1 & $\begin{array}{l}\text { Chipe } \\
\text { Minuope }\end{array}$ & $\begin{array}{l}\text { Andrés } \\
\text { Murillo }\end{array}$ & Quevedo & 150 & $\begin{array}{l}\text { Muy } \\
\text { alto }\end{array}$ \\
\hline P2 & $\begin{array}{l}\text { Chipe } \\
\text { Minuope }\end{array}$ & Johnny Moran & Quevedo & 200 & $\begin{array}{l}\text { Muy } \\
\text { alto }\end{array}$ \\
\hline P3 & La Cadena & Alfredo Vera & Quevedo & 50 & $\begin{array}{c}\text { Modera } \\
\text { do }\end{array}$ \\
\hline P4 & Fruta de Pan & Olmedo Chan & Quevedo & 150 & $\begin{array}{l}\text { Muy } \\
\text { alto }\end{array}$ \\
\hline P5 & Lechugal & Wilson Ochoa & Quevedo & 700 & $\begin{array}{l}\text { Muy } \\
\text { alto }\end{array}$ \\
\hline P6 & $\begin{array}{l}\text { San } \\
\text { Francisco }\end{array}$ & $\begin{array}{l}\text { Ricardo } \\
\text { Montero }\end{array}$ & Quevedo & 200 & $\begin{array}{l}\text { Muy } \\
\text { alto }\end{array}$ \\
\hline P7 & El Guayacán & $\begin{array}{c}\text { Efrain } \\
\text { Villarreal }\end{array}$ & Quevedo & 1600 & $\begin{array}{l}\text { Muy } \\
\text { alto }\end{array}$ \\
\hline P8 & Bombón & Paola Albán & Quevedo & 150 & $\begin{array}{l}\text { Muy } \\
\text { alto }\end{array}$ \\
\hline P9 & Pichilingue & Jaime López & Quevedo & 300 & $\begin{array}{l}\text { Muy } \\
\text { alto }\end{array}$ \\
\hline P10 & $\begin{array}{l}\text { INIAP } \\
\text { Pichilingue }\end{array}$ & INIAP & Quevedo & 250 & $\begin{array}{l}\text { Muy } \\
\text { alto }\end{array}$ \\
\hline P11 & San Pablo & $\begin{array}{c}\text { Nixon } \\
\text { González }\end{array}$ & Babahoyo & 100 & $\begin{array}{c}\text { Modera } \\
\text { do }\end{array}$ \\
\hline P12 & Palmar & Euterio Quiroz & $\begin{array}{l}\text { Babahoyo } \\
\text { (CEDEGE) }\end{array}$ & 100 & $\begin{array}{c}\text { Modera } \\
\text { do }\end{array}$ \\
\hline P13 & Cedral 2 & Rafael Bolívar & $\begin{array}{l}\text { Babahoyo } \\
\text { (CEDEGE) }\end{array}$ & 600 & $\begin{array}{l}\text { Muy } \\
\text { alto }\end{array}$ \\
\hline P14 & La Legua & Efrén Germán & Babahoyo & 50 & $\begin{array}{c}\text { Modera } \\
\text { do }\end{array}$ \\
\hline P15 & La Legua & Luis Campo & Babahoyo & 50 & $\begin{array}{c}\text { Modera } \\
\text { do }\end{array}$ \\
\hline P16 & Pimocha & $\begin{array}{l}\text { Alejandro } \\
\text { Campo }\end{array}$ & Babahoyo & 250 & $\begin{array}{l}\text { Muy } \\
\text { alto }\end{array}$ \\
\hline P17 & Pimocha & Ramón Morán & Babahoyo & 150 & $\begin{array}{l}\text { Muy } \\
\text { alto }\end{array}$ \\
\hline
\end{tabular}

Lombeida García E, Gómez PandoL, Reyes Borja WO, Triviño Gilces C, Hasang Moran E, Duran Canare PL, Hufana Duran D. Densidad de población de Meloidogyne graminicola en plantaciones comerciales de arroz y comportamiento de líneas avanzadas en la provincia de Los Ríos, 
Rev. Soc. cient. Parag. 2021;26(1):64-81

\begin{tabular}{clcccc}
\hline P18 & $\begin{array}{l}\text { Mata de } \\
\text { Cacao } \\
\text { Cacharí }\end{array}$ & $\begin{array}{c}\text { Juan } \\
\text { Contreras } \\
\text { Miguel } \\
\text { Pambrano }\end{array}$ & Babahoyo & 300 & $\begin{array}{c}\text { Muy } \\
\text { alto } \\
\text { Modera } \\
\text { do }\end{array}$ \\
P20 & Cacharí & $\begin{array}{c}\text { Walson Loor } \\
\text { Wilsono } \\
\text { do }\end{array}$ & Babahoyo & 50 & \begin{tabular}{c} 
Modera \\
\hline
\end{tabular}
\end{tabular}

En la Tabla 5, se presentan los resultados de las poblaciones de $M$. graminicola que se obtuvieron en $10 \mathrm{~g}$ de raíces, en los 20 sitios de muestreo. De acuerdo con la información obtenida en el muestreo de raíces realizado en el cantón Quevedo, recinto Pichilingue, se observa una población con un valor de 70000 J2, clasificada de acuerdo con la escala como muy alta; mientras que en el cantón Babahoyo, en el sector CEDEGE se obtuvo la mayor población de M. graminicola, con 28000 J2, también calificada como muy alta.

Según estudios realizados por Bridge y Page en 1982 (13), 4000 individuos de M. graminicola por planta, pueden causar pérdidas en la producción de arroz y tiene que ver mucho con la etapa de desarrollo de la planta. Después del trasplante, $1000 \mathrm{~J} 2 /$ planta pueden reducir la altura, número de macollos y la producción hasta en un 6 por ciento. Resultados previos han evidenciado la elevada presencia de M. graminicola, en zonas arroceras de Ecuador.

Los resultados del presente estudio muestran que existen altas poblaciones de M. graminicola en suelo y raíces en las diferentes provincias arroceras del Ecuador, encontrándose las poblaciones más altas en los cantones de Babahoyo, Quevedo y Valencia, pertenecientes a la provincia de Los Ríos (11). Una de las probables causas de la elevada ocurrencia del nematodo en los campos comerciales, es el monocultivo de arroz con cultivares susceptibles a lo largo de muchos años, lo que ha contribuido significativamente al rápido crecimiento poblacional de este nematodo en el suelo. Existen aparentemente dificultades para hacer rotación de cultivos con plantas no hospederas. Otra causa probablemente se relaciona con el desconocimiento y la falta de diagnóstico del problema, por técnicos y productores, debido a que los daños causados por M. graminicola, pueden ser confundidos con otros síntomas, tales como: deficiencias nutricionales, ataque de otros patógenos del suelo, fitotoxicidad causada por los herbicidas o por daños indirectos como el exceso de hierro en el suelo.

Lombeida García E, Gómez PandoL, Reyes Borja WO, Triviño Gilces C, Hasang Moran E, Duran Canare PL, Hufana Duran D. Densidad de población de Meloidogyne graminicola en plantaciones comerciales de arroz y comportamiento de líneas avanzadas en la provincia de Los Ríos, 
Rev. Soc. cient. Parag. 2021;26(1):64-81

Tabla 5. Densidad poblacional de M. graminicola determinada en 10 gramos de raíces de arroz, procedentes de plantaciones comerciales de los cantones Babahoyo y Quevedo.

\begin{tabular}{|c|c|c|c|c|c|}
\hline Código & Recinto & Propietario & Zona & $\begin{array}{c}M . \\
\text { graminicola } \\
/ 10 \mathrm{~g} \text { de } \\
\text { raíces }\end{array}$ & Rangos \\
\hline P1 & Chipe Minuope & $\begin{array}{l}\text { Andrés } \\
\text { Murillo }\end{array}$ & Quevedo & 11500 & Muy alto \\
\hline P2 & Chipe Minuope & Johnny Moran & Quevedo & 3000 & Muy alto \\
\hline P3 & La Cadena & Alfredo Vera & Quevedo & 10500 & Muy alto \\
\hline P4 & Fruta de Pan & Olmedo Chan & Quevedo & 7500 & Muy alto \\
\hline P5 & Lechugal & Wilson Ochoa & Quevedo & 17500 & Muy alto \\
\hline P6 & San Francisco & $\begin{array}{l}\text { Ricardo } \\
\text { Montero }\end{array}$ & Quevedo & 6000 & Muy alto \\
\hline P7 & El Guayacán & $\begin{array}{l}\text { Efraín } \\
\text { Villarreal }\end{array}$ & Quevedo & 17000 & Muy alto \\
\hline P8 & Bombón & Paola Albán & Quevedo & 50000 & Muy alto \\
\hline P9 & Pichilingue & Jaime López & Quevedo & 70000 & Muy alto \\
\hline P10 & $\begin{array}{l}\text { INIAP } \\
\text { Pichilingue }\end{array}$ & INIAP & Quevedo & 6000 & Muy alto \\
\hline P11 & San Pablo & $\begin{array}{l}\text { Nixon } \\
\text { González }\end{array}$ & Babahoyo & 3500 & Muy alto \\
\hline P12 & Palmar & Euterio Quiroz & $\begin{array}{l}\text { Babahoyo } \\
\text { (CEDEGE) }\end{array}$ & 28000 & Muy alto \\
\hline P13 & Cedral 2 & Rafael Bolívar & $\begin{array}{l}\text { Babahoyo } \\
\text { (CEDEGE) }\end{array}$ & 28000 & Muy alto \\
\hline P14 & La Legua & Efrén Germán & Babahoyo & 3000 & Muy alto \\
\hline P15 & La Legua & Luis Campo & Babahoyo & 3500 & Muy alto \\
\hline P16 & Pimocha & $\begin{array}{l}\text { Alejandro } \\
\text { Campo }\end{array}$ & Babahoyo & 9000 & Muy alto \\
\hline P17 & Pimocha & Ramón Morán & Babahoyo & 2000 & Muy alto \\
\hline P18 & Mata de Cacao & $\begin{array}{l}\text { Juan } \\
\text { Contreras }\end{array}$ & Babahoyo & 9000 & Muy alto \\
\hline P19 & Cacharí & $\begin{array}{l}\text { Miguel } \\
\text { Zambrano }\end{array}$ & Babahoyo & 4500 & Muy alto \\
\hline P20 & Cacharí & Wilson Loor & Babahoyo & 4500 & Muy alto \\
\hline
\end{tabular}

Lombeida García E, Gómez PandoL, Reyes Borja WO, Triviño Gilces C, Hasang Moran E, Duran Canare PL, Hufana Duran D. Densidad de población de Meloidogyne graminicola en plantaciones comerciales de arroz y comportamiento de líneas avanzadas en la provincia de Los Ríos, 


\section{Comportamiento de líneas avanzadas de arroz a M. graminicola provenientes de raíces y suelos}

\section{Densidad poblacional de $M$. graminicola en raíces, en líneas avanzadas F5 de arroz.}

El análisis de varianza de la densidad poblacional M. graminicola en raíces, muestra que existe alta significancia estadística entre los tratamientos; es decir, una respuesta diferente entre las líneas avanzadas $\mathrm{F}_{5}$ evaluadas. En la Figura1, se muestran los resultados y se aprecia que el rango varió de 5530 a $63210 \mathrm{~J} 2 / 10 \mathrm{~g}$ de raíces; correspondiendo el menor valor a la línea 32 (PUYÓN/JP002 P11-10 P74) y el mayor valor a la línea 7 (PUYÓN/JP002 P8-29 P8-5). El experimento tuvo un valor promedio de $22888 \mathrm{~J} 2$ /10 g de raíces y el testigo comercial INIAP 15 (susceptible), tuvo un valor promedio de 17190 J2 /10 g de raíces.

Sin embargo, las líneas 32 (PUYÓN/JP002 P11-10 P74); 5 (PUYÓN/JP002 P8-20 P13); 31 (PUYÓN/JP002 P11-10 P22) y 2 (PUYÓN/JP002 P8-20 P14), presentaron los valores promedio poblacionales más bajos en el orden descrito a continuación 5530, 7130, 7590 y 8080 J2 /10 g de raíces. En contraste, las más susceptibles a $M$. graminicola fueron las líneas 7 (PUYÓN/JP002 2 P8-29 $\quad$ P8-5); 8 (PUYÓN/JP002 P8-29 $\quad$ P8-16); 12 (PUYÓN/JP002 P8-30 P55-2) y 10 (PUYÓN/JP002 P8-29 P49-30), con los promedios de 63210, 50060, 43750 y 43310 J2 /10 g de raíces; respectivamente.

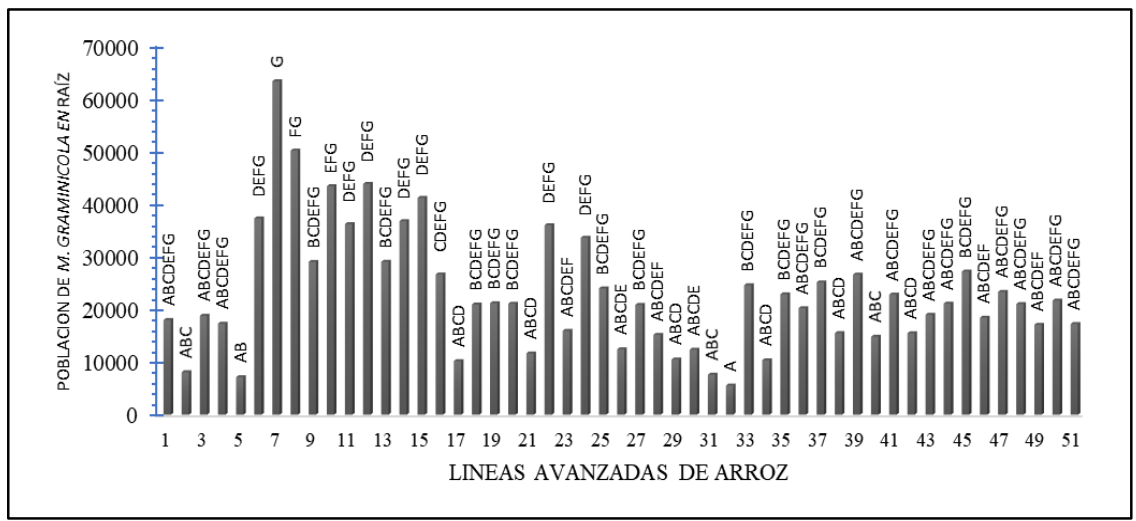

Figura 1. Densidad poblacional de M. graminicola (J2 /10 g de raíces), en líneas avanzadas $\mathrm{F}_{5}$ de arroz.

Lombeida García E, Gómez PandoL, Reyes Borja WO, Triviño Gilces C, Hasang Moran E, Duran Canare PL, Hufana Duran D. Densidad de población de Meloidogyne graminicola en plantaciones comerciales de arroz y comportamiento de líneas avanzadas en la provincia de Los Ríos, 


\section{Densidad poblacional de M. graminicola en suelo en líneas avanzadas $F_{5}$ de arroz.}

El resultado del analisis de la varianza de esta variable, muestra alta significancia estadistica entre los tratamientos. El rango observado varió de 27 a $2960 \mathrm{~J} 2$ en $100 \mathrm{~cm}^{3}$ de suelo (Figura 2). El valor más bajo corresponde a la línea 24 (PUYÓN/JP002 P8-28 P28) y el más alto a la línea 36 (JP003/JP001 P169 P23-6-GL).

Se identificaron las líneas que presentaron los menores valores, tales como las líneas 24, 28 y 30, con promedios de 27, 29 y 31 J2 en $100 \mathrm{~cm} 3$ de suelo respectivamente. Esto contrasta con las líneas que obtuvieron los valores más altas, siendo las líneas 36, con valores promedios de 2960 y 2510 en $100 \mathrm{~cm}^{3}$ de suelo respectivamente. Las líneas derivadas de cruces interespecíficos entre Oryza rufipogon G. (PUYÓN) x Oryza sativa L. ssp. japónica, mostraron menores valores en general.

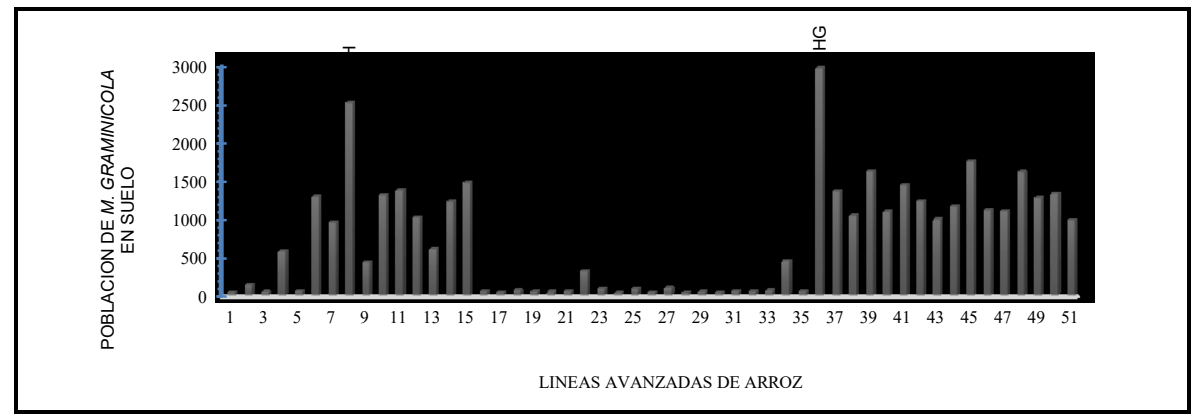

Figura 2. Densidad poblacional de M. graminicola (J2 /100 $\mathrm{cm}^{3}$ de suelo), en líneas avanzadas $\mathrm{F}_{5}$ de arroz.

\section{Índice de reproducción de $M$. graminicola en líneas avanzadas $F_{5}$ de arroz.}

En lo que concierne al índice de reproducción de $M$. graminicola, el rango varió de 2 a 25 veces. La línea en la que se observe un menor índice de reproducción fue la línea 32 (PUYÓN/JP002 P11-10 P74), considerada como un hospedero eficiente, estando en el rango de 1.1 a 2, de acuerdo con la escala determinada por CIP (1985). Todas las demás líneas según la escala mencionada se calificaron como hospederos muy eficientes. De igual 
modo, se detectaron líneas en las cuales se encontraron mayores índices de reproducción del nematodo, tales como las líneas 7 (PUYÓN/JP002 P8 -29 P8-5), 8 (PUYÓN/JP002 P8-29 P8-16), 12 (PUYÓN/JP002 P8-30 P55-2) y 10 (PUYÓN/JP002 P8 -29 P49-30) que presentaron valores de reproducción de 25, 20, 18 y 17 veces, respectivamente; en la variedad INIAP 15 (susceptible a M. graminicola), se observó un índice de reproducción del nematodo igual a 7.

Algunas de las líneas derivadas de cruces interespecíficos entre Oryza rufipogon G. (PUYÓN) x Oryza sativa L. ssp. japonica, se encontró menores valores de índices de reproducción del nematodo, comparados con el valor de reproducción en la variedad comercial INIAP 15. Igualmente, los valores obtenidos en las líneas derivadas de cruces intraespecíficos de Oryza sativa L. ssp. japonica, se mantienen en índices de reproducción mayores a 2; sin embargo, aunque sean menores, siguen siendo hospederos eficientes. Lo cual indican que el índice de reproducción del nematodo se usa para identificar la resistencia en el material genético. Esto relaciona la población final con la inicial y refleja el nivel de incremento del nematodo, considerándose a una planta resistente cuando el valor del índice es menor a 1, y susceptible cuando el índice es mayor este valor.

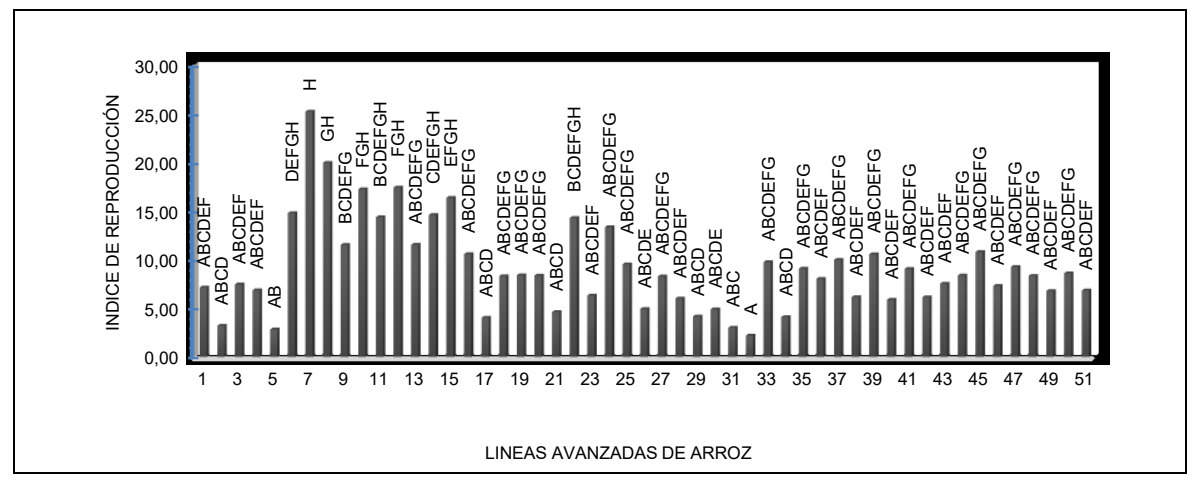

Figura 3. Índice de reproducción de M. graminicola en líneas avanzadas $F_{5}$ de arroz.

Los genotipos estudiados fueron susceptibles a M. graminicola según la escala valorada por Ramos; sin embargo, se observó la línea 32 (PUYÓN/JP002 P11-10 P74) que proviene de cruces interespecíficos con la menor cantidad de nematodos en $10 \mathrm{~g}$ de raíces comparado con el testigo comercial INIAP-15 (12). Probablemente este material genético tiene 
resistencia cuantitativa, asociada con la capacidad de reducir la población del nematodo, pero con síntomas de susceptibilidad.

Uno de los síntomas visibles que mostraron las líneas infestadas con $M$. graminicola fue el amarillamiento de hojas a los 35 días después de la inoculación. Esto puede estar relacionado a que la infección o daño causado por los nematodos en las raíces de las plantas de arroz, interfiere de manera directa en el flujo de absorción y transporte de agua y nutrientes en la planta, originando síntomas en la parte foliar tales como raquitismo, clorosis y pérdida de vigor(16).

En esta investigación, la alta incidencia de M. graminicola en las raíces de las plantas de algunas líneas avanzadas $\mathrm{F}_{5}$ de arroz, corrobora lo expresado por Reyes, que menciona que los hospederos favoritos de los nematodos son las gramíneas especialmente el $\operatorname{arroz}^{(17)}$.

Determinada la densidad poblacional de M. graminicola en $100 \mathrm{~cm}^{3}$ de suelo, es importante destacar que, las líneas avanzadas F5 derivadas de cruces interespecíficos entre Oryza rufipogon G. (PUYÓN) x Oryza sativa L. ssp. japónica, mostraron menores valores en general, comparados con los valores obtenidos en las líneas provenientes de cruces intraespecíficos de Oryza sativa L. ssp. japonica, observado a través de la aplicación de la escala de severidad y incidencia de Ramos, para calificar la respuesta a Meloidogyne en arroz ${ }^{(12) .}$

\section{CONCLUSIONES}

La línea 32 (PUYÓN/JP002 P11-10 P74) presentó la menor cantidad de nematodos en $10 \mathrm{~g}$ de raíces comparado con el testigo comercial INIAP-15. La línea con menor índice de reproducción fue la línea 32 (PUYÓN/JP002 P11-10 P74), considerada como un hospedero eficiente. Las líneas restantes obtuvieron valores mayores a 2, definiéndose como hospederos muy eficientes.

Las líneas que obtuvieron las menores densidades poblacionales de $M$. graminicola en raíces, deben ser evaluadas por su potencial de rendimiento y determinar el nivel de daño por el nematodo comparadolas con variedades susceptibles.

Lombeida García E, Gómez PandoL, Reyes Borja WO, Triviño Gilces C, Hasang Moran E, Duran Canare PL, Hufana Duran D. Densidad de población de Meloidogyne graminicola en plantaciones comerciales de arroz y comportamiento de líneas avanzadas en la provincia de Los Ríos, 


\section{REFERENCIAS}

1. Golden A, Birchfield W. Rice root-knot nematode Meloidogyne graminicola as a new pest of Rice. Pl Dis Reptr.1968;52:423.

2. Velásquez V. Economic, social and political analysis of the agri-food chain of rice in Ecuador, period 2005-2014. Dissertation prior to obtaining the title of Economist. Catholic University of Ecuador. Faculty of Economics, Quito-Ecuador. 2016:143.

3. Díaz D, Chaparro A. Methods and agricultural uses of genetic engineering applied to rice. Rev. Colomb. Biotecnol. 2012;14(2):179-195.

4. Franquet J. Varieties and rice improvement. In: Universitat Internacional de Catalunya. 2004. 157:199. (in Spanish)

5. Acevedo M, Castrillo W, Belmonte U. Origin, evolution, and diversity of rice. Agronomía Trop. 2006;56(2):151-170.

6. Bridge J, Star J. Plant nematodes of agriculture importance: A color Handbook Migratory Endoparasite. Londres. Academic Press. 2007;5759.

7. Soriano I, Prot J, Matias D. Expression of tolerance for Meloidogyne graminicola in rice cultivars as affected by soil type and flooding. Journal of Nematology.2000; 32(3):309 - 317.

8. Talavera M. Manual de Nematologia Agrícola. Introducción al análisis y al control nematológico para agricultores y técnicos de agrupaciones de defensa vegetal. 2003;23. Available in: http://www.caib.es/sacmicrofront/archivopub.do?ctrl=CNTSP722ZI456 9\&id $=4569$

9. Institute of Agricultural and Fisheries Research and Training. (SERVIFAPA). Integrated Management of Phytoparasite Nematodes in Horticultural Crops. Editors: Board of Santa Lucia. Spain. 2014, 120 p.

10.INAMHI. National Institute of Meteorology and Hydrology. Agrometeorology Station of the Faculty of Agricultural Sciences of the Technical University of Babahoyo, Los Ríos, Ecuador; 2019. comerciales de arroz y comportamiento de líneas avanzadas en la provincia de Los Ríos, 
11. Triviño C, Navia D, Velasco L. Guide to recognizing root damage and methods of sampling and extraction of nematodes in roots and soil. Yaguachi, Ecuador. Autonomous National Agricultural Research Institute. Litoral Sur Experimental Station "Dr. Enrique Ampuero Pareja". Disclosure Bulletin. 2013;43:3-17.

12. Ramos J, Franco J, Ortuño N, Oros R, Main G. Incidence and Severity of Nacobbus aberrans and Globodera spp. in the cultivation of potatoes in Bolivia: losses in the gross value of its production. Cochabamba, Ibta/Proimpa. 1998;201.

13. Bridge J, Page S. The rice root-knot nematode, Meloidogyne graminicola, on deep water rice (Oryza sativa L. ssp. indica). Revue de Nématologie. 1982;5:225-232

14.Padgham J, Duxbury J, Mazid A, Abawi G, Hossain H. Yield los caused by Meloidogyne graminicola on low-land rainfed rice in Bangladesh. J Nematol. 2004;36:42-48.

15. Triviño C, Navia F, Velasco L. Plant - Parasitic Nematodes Associated with rice in Ecuador. Nematropica. 2016;46(1):45-53

16. Hettwer K, Jähne M, Frost K, Giersberg M, Kunze G, Trimborn M, Reif J Türk M Gehrmann L, Dardenne F. Validation of Arxula Yeast Estrogen Screen assay for detection of estrogenic activity in water samples: results of an international interlaboratory study. Sci. Total Environ. 2018;621:612-625.

17. Reyes I. Determination of the susceptibility of forty rice materials (Oryza sativa L.) to Meloidogyne graminicola. Agricultural Engineer thesis. Technical University of Babahoyo. Ecuador. Faculty of Agricultural Sciences. 2013:21. 